\title{
A new method to estimate the lifetime of long life product categories
}

\author{
Cyrille F. Dunant ${ }^{\mathrm{a}}$, Trishla Shah ${ }^{\mathrm{a}}$, Michał P. Drewniok ${ }^{\mathrm{a}}$, Matteo Craglia ${ }^{\mathrm{a}}$, Jonathan M. \\ Cullen, ${ }^{\mathrm{a}, *}$ \\ ${ }^{a}$ University of Cambridge, Cambridge, UK
}

\begin{abstract}
Increased recycling and reuse rates are a central part of the objectives laid out by the COP21 (?). Nonetheless, the practical implementation of what has been called the circular economy, as well as its true potential are not easily established. This is because the impact and implementation time scales of any intervention depend on knowing the lifetime of products, which is frequently unknown. This is particularly true in construction, responsible for $39 \%$ of worldwide emissions, $11 \%$ of which are embodied.

Most MFA models will simply assume a range of plausible life expectancies when bottom-up data is lacking (e.g. for buildings (?)). In this work, we propose a novel method of identification using the high quality but highly aggregated trade data available, and use it to establish a "mortality curve" for buildings and other long-lasting products. This identification method is intended to provide more reliable inputs to existing MFA models. It is widely applicable due to the general availability of the underlying data. Using it on UK trade data, we identify product classes at 1 year for packaging/home scrap, one around 10 years for vehicles/equipment, and around 50 years for construction. The identification approach was then validated using classical approaches using bottom up data for vehicles.
\end{abstract}

${ }^{*}$ Corresponding author

Preprint submitted to Elsevier

today 


\section{Motivation}

Buildings and the construction industry are responsible for almost $39 \%$ of energyrelated carbon dioxide emissions and 36\% of global energy use (?). A quarter of these emissions are connected to steel and cement used in construction, 4 and 7\% global emissions respectively (??). Stocks, and to a large extent inflows, of materials have stabilised in the 'industrialised' countries since the 1970s, but this is not yet the case for outflows (?). In turn, the outflows are a critical aspect of determining the emissions of future inflows, due to changing recycled content. In the less industrialised parts of the world, urbanisation growth (?) leads to the prediction of a doubling of the building floor area from 2015 to 2050 , from $223 \cdot 10^{9} \mathrm{~m}^{2}$ to $415 \cdot 10^{9} \mathrm{~m}^{2}$. Projections using material flow analysis rely on demographic and economic forecasts as well as assumptions about the life expectancy of buildings. Buildings are only one of a number of long-life 'products' for which there are no fully satisfactory life expectancy models. Yet these products represent an important part of the overall flow of steel, and their eventual retirement will impact the availability of material for recycling with important consequences for the steel industry (?).

A survey of available data indicates that the uncertainty around estimates of building life expectancy is considerable. The ? indicates buildings should be designed for a lifetime of 60 years. However, real life spans can be much shorter as confirmed in many surveys. The Minnesota Demolition Survey (?) covered demolished buildings in St. Paul between 2000 to mid-2003 and reported that from a pool of 227 buildings, $6 \%$ and $23 \%$ were demolished at 0-25 and 25-50 years, respectively. Only $16 \%$ (17 out of 104) of the commercial buildings were demolished due to the "building's physical condition". The US construction codes have changed considerably between the construction of these buildings and now: current ACI codes suggest a 50 year baseline for concrete buildings. ? analysed a dataset of all 50,818 buildings demolished in Finland between 2000 and 2012, showing that non-domestic buildings have typical life spans of less than 40 years, which 
is considerably shorter than domestic buildings, as shown in Table 1. Good data is also available in Japan: ? distinguished the lifetime distributions of buildings in Japan by construction material, and ? could assess the impact of economic prosperity on building lifetimes. In general, such high quality statistics are not available, and it is not correct to infer life time of buildings from the prescription of building codes.

\begin{tabular}{ll} 
Table 1: Average age at the time of demolition by building type \\
\hline Building type & Lifetime \\
\hline Detached houses & 64 \\
Terraced (row) houses & 44 \\
Block of flats & 62 \\
Dormitories & 36 \\
Holiday cottages & 47 \\
Utility buildings & 47 \\
\hline Commercial and office buildings & 39 \\
Public buildings & 41 \\
Warehouses & 37 \\
Industrial buildings & 37 \\
Agricultural buildings & 35 \\
Transport buildings & 36 \\
Other buildings & 32 \\
\hline
\end{tabular}

The high levels of uncertainty for long-lived products propagates to the analysis of the material flows. The same product category can have very different life-times depending on the country or circumstances, which makes it problematic to use data obtained in one area to another. Table 2 presents lifetimes of steel for Sweden, Japan and the UK. Lifetimes are calculated by ? and ? by applying lifetime estimates of the the steel consumption for the year when end-of-life (EOL) scrap was collected. Meanwhile, ? conducted volume correlation to calculate lifetimes of Eol steel, ? used Dynamic Material Flow Analysis (DFMA) to estimate lifetimes for stainless steels in Japan, and ? used DFMA to assess service lifetimes of EOL steel. The range of results highlights the need for a top-down approach which can identify the lifetimes of long-lived products in the absence of good bottom-up data. 
Table 2: Lifetime analysis of steel

\begin{tabular}{lllll}
\hline Authors & Country & Sector & Year & Lifetime \\
\hline $\boldsymbol{?}$ & UK & Construction & 2000 & 60 \\
$\boldsymbol{?}$ & Sweden & Construction & 2006 & 80 \\
$\boldsymbol{?}$ & Japan & Stainless steel for construction & 2005 & 30 \\
$\boldsymbol{?}$ & UK & Lifetime of total steel & 2000 & 31 \\
$\boldsymbol{?}$ & Sweden & Lifetime of total steel & 2006 & 35 \\
$\boldsymbol{?}$ & Sweden & Service lifetimes of EOL steel & 2012 & 35 \\
$\boldsymbol{?}$ & World & Service lifetimes of EOL steel & 2012 & 28 \\
$\boldsymbol{?}$ & World & Service lifetimes of products & 2013 & various \\
$\boldsymbol{?}$ & World & Service lifetimes of products & 2014 & various \\
\hline
\end{tabular}

Many studies rely on the design life of products to infer the end-of-life distribution because of the lack of reliable data (???), but there are some exceptions: there is good commercial data available in many countries for cars, and lifetime estimation by the fitting of a single Weibull distribution has yielded good results (???). Current methods for identifying the life-time distribution of goods have a fixed number of parameters: they attempt to identify the parameters of a single distribution. But it is in fact not known whether this is enough for any particular class of product, and in particular in the case of buildings, which can differ widely depending on function, location, design, etc. The fundamental assumption behind the identification process in the previous works is that a class of product has a characteristic life-time, modulated by a shape parameter (typically using a Weibull distribution). In this work, we identify product categories, after the identification which tries to capture accurately narrow life-time categories instead of product classes as such.

A source of data which could be used to estimate lifetimes are trade statistics on the basic inputs for buildings and long lasting goods — steel and concrete — which have been collected in many countries since the early $20^{\text {th }}$ century. These high quality data series, however, lack disaggregation into finished product categories or uses. The steel trade statistics (?) also tabulate scrap as a proxy for outflows. In principle it is possible, given the inflows and outflows, to compute a transfer function (TF) which describes the 
probability distribution of the delay between an inflow and its release as an outflow, following use and discard. A key difficulty is that not all steel is accounted for in the scrap based outflow calculations, because of imperfect accounting, so-called hibernating steel stocks and landfilling. Therefore, a TF derived from inflows and outflows, cannot quantitatively describe the amount of steel which goes into any particular product category, and is therefore not a lifetime probability distribution function (PDF).

This shortcoming is less important than it may seem: in most instances, the recycling rates are known - e.g. in the uK, $7 \%$ of heavy structural sections and tubes, $15 \%$ of steel piles (sheet and bearing), and $10 \%$ of profile steel cladding in construction is currently reused (??), and almost all other steel is recycled. Therefore, the total amounts can be obtained from other data sources and the TF can be transformed into a PDF.

In this paper we present the method and an overall TF for steel in the UK. We show how to extract individual TFs for buildings and cars and produce the PDF from them. We then compare our top-down method with bottom-up data for these two product categories, to validate the derived PDF. Such a method, as proposed in this paper, can then be used to provide inputs into more general MFA models such as the ones from ? or $?$.

\section{Methods}

In this section, we detail how the TF is derived and fitted to inflow and outflow data. Then, a method is described for validating the TF against bottom-up data for building and vehicles.

\subsection{The Transfer Function}

The TF describes how each unit of steel input, used for indirect sources (i.e. cars) or direct sources (i.e. beams) is output to scrap. This analysis considers each unit of steel to include all product categories, amalgamating all uK steel consumption and scrap generation. The function is equivalent to a lifetime probability distribution describing 
the delay between an input of steel into the use-phase and its corresponding output as scrap, considering the fraction lost ether to landfill or kept forever in stocks.

The predicted scrap output (output) from the steel input into the production of goods ( input) is:

$$
\text { output }=\mathrm{TF} * \text { input }
$$

where $\mathrm{TF}$ is the $\mathrm{TF}$ and $*$ is the convolution operator.

$$
\text { output }=\mathrm{TF} * \text { input }=\int_{0}^{t} \mathrm{TF}(\tau) \operatorname{input}(t-\tau) \mathrm{d} \tau
$$

We have investigated two variants: a sum of Weibull distributions; a sum of lognormal distributions. Weibull and log-normal distributions are good choices to model lifetimes as they are semi-infinite, easily shaped and have been shown experimentally to provide a good fit to lifetime data (?). Additionally, to account for the fact that both distribution types have a probability of 0 at $t=0$, a decaying exponential was included in the TF to reflect any scrap that is generated within the first year. This could be scrap from short-life steel products, such as packaging containers, or due to premature failures of long-life products. To account for imperfect recovery and allow some proportion of steel to be permanently accumulated in the system, for example, a loss fraction $\phi_{\text {loss }}$ was determined in the fitting procedure, such that the integral of the final TF was equal to one minus this fraction. This accumulation of steel is assumed to remain permanently in the stock (hibernating), or to be lost (i.e. to landfill) with no expectation of recovery.

The general shape of the TF, given distributions $\mathcal{D}_{i}$ is:

$$
\mathrm{TF}=\left(\phi_{1} e^{-t}+\sum_{i} \phi_{i} \mathcal{D}_{i}(t)\right)\left(1-\phi_{\text {loss }}\right)
$$

where the $\phi$ values are the unknown parameters which determine the dominant characteristic times in the overall TF. 
The parameters in the sum of log-normal distributions (equation 4) are $\sigma$, the spread of each distribution; $\mu$, the characteristic time; and $\phi$ the fraction by which each distribution is weighted. For each element in the summation, the time parameter $\mu$ is set at regular 5-year intervals while the spread $\sigma$ is set constant at 5 years.

$$
\mathrm{TF}=\left(\phi_{1} e^{-t}+\sum_{i} \phi_{i} \exp \left\{-\frac{\left(\log (t)-\log \left(\mu_{i}\right)\right)^{2}}{\sigma_{i}^{2}}\right\} \frac{\sigma_{i} \sqrt{2 \pi}}{t}\right)\left(1-\phi_{\mathrm{loss}}\right)
$$

The parameters for the TF based on the Weibull distribution (equation 5) were defined in a similar way to set the characteristic lifetimes at the same intervals with the same spread as described for the log-normal distribution. This was achieved by defining the shape parameter $\beta$, the scale parameter $\eta$ and the location parameter $\gamma$. For example, for five-year intervals $\beta=3.1$;

$$
\mathrm{TF}=\left(\phi_{1} e^{-t}+\sum_{i} \phi_{i} \exp \left\{-\frac{\left(t-\gamma_{i}\right)^{\beta_{i}}}{\eta_{i}}\right\} \frac{\beta_{i}}{\eta_{i}}\left(\frac{t-\gamma_{i}}{\eta_{i}}\right)^{\beta_{i}}\right)\left(1-\phi_{\mathrm{loss}}\right)
$$

As well as investigating the use of the Weibull distribution versus the log-normal, the characteristic times were also varied between runs to identify the optimal interval between adjacent peaks. The sampling rates chosen were: $1,2,5,7$, and 14 years. If the discretisation is too fine, the function is not useful as the scrap market is sensitive to variations in socio-economic factors which we do not wish to model explicitly. On the other hand, if the intervals are too wide, the uncertainty in the results would be greater and a smoothing effect is likely to occur. The sampling rates chosen are odd so the peaks are neatly centered on data points, except for 2 , which is the minimum theoretical sampling rate which could identify the transfer function and 14 , which is a "large" value corresponding to the commonly assumed life-time of cars.

To identify these functions meaningfully, they must satisfy the following requirements:

- The function must be easily parametrised as too many parameters make the fit with the limited data meaningless. 
- The parameters to be determined in the fitting process must have meaning such that the result can be interpreted.

- The function must satisfy the appropriate boundary conditions. For example, a sum of Gaussian functions would be unsuitable as they extend from $-\infty$ to $+\infty$, allowing negative lifetimes to have a non-zero probability.

- The fit procedure must yield a unique answer.

The parametrisation chosen in this study satisfies all of the above conditions. A sum of either log-normal or Weibull distributions meets the boundary condition requirements and can be easily parametrised. Therefore, this analysis explores both distribution types.

The transfer function identified for any product category is not composed of a single peak, but multiple ones, allowing for a large flexibility in the shape of the fitted function.

\subsection{The lifetime probability distribution}

From the transfer function for a specific product class, identified using the procedure described in 2.3, it is possible to derive a lifetime probability distribution function (PDF). The peaks corresponding to the product class are isolated from the global TF and need to be scaled to correspond to life-times. The TF corresponding to the product modelled by the peaks $i=i_{0} \ldots i_{N}$ is:

$$
\mathrm{TF}=\text { recycling rate } \cdot \sum_{i=i_{0}}^{i=i_{N}} \phi_{i} \mathcal{D}_{i}(t)
$$

This requires further inputs concerning the product class: the recycling rate at end-of-life. These data are typically available from trade bodies or official statistics. To transform the TF into a PDF, the following normalisation is needed:

$$
\mathrm{PDF}=\frac{\mathrm{TF}}{\int_{0}^{\infty} \mathrm{TF} \mathrm{d} t}
$$

The lifetime probability distribution is then what is used as input in MFA models. We demonstrate how to use the TF to derive the PDF for two classes of products, buildings and cars in the result section below. 
A difficulty arises when multiple product classes have TF with overlapping peaks. This can be resolved knowing the amount of steel going into the production of the products, information commonly available from trade bodies. In the case of two products $A$ and $B\left(\mathrm{TF}_{A, B}\right.$ the identified overall $\left.\mathrm{TF}\right)$ :

$$
\mathrm{TF}_{A, B}=\frac{\text { input }_{A} \cdot \text { recycling rate }_{A} \cdot \mathrm{TF}_{A}+\text { input }_{B} \cdot \text { recycling rate }_{B} \cdot \mathrm{TF}_{B}}{\text { input }_{A}+\text { input }_{B}}
$$

Because the inputs and recycling rates are typically known, and since in the overlapping section at every peak, ( $c$ a constant):

$$
\mathrm{TF}_{A}=c \cdot \mathrm{TF}_{B} \quad c=\frac{\text { input }_{B} \cdot \text { recycling rate }}{B}
$$

We can reduce the problem to a single unknown, e.g. for $B$ :

$$
\mathrm{TF}_{B}=\mathrm{TF}_{A, B} \frac{\text { input }_{A}+\text { input }_{B}}{\text { input } \left._{A} \cdot \text { recycling rate }_{A} \cdot c+\text { recycling rate }_{B}\right)}
$$

To properly identify the PDF, the fitting procedure should be done over a period where it does not change too much. Otherwise the identified PDF will be a mixture, and much broader than the actual distribution at any point in time. Building life-times are relatively stable, unlike car life-times and this is visible at the bottom of Figure 1 where the choice of the fitting period affects the observed distribution.

\subsection{The fitting procedure}

A complete definition of the TF involves determining the unknowns in equations 4 and 5: the fractions by which each element is weighted $\phi$; the constant fraction of scrap which persists in the system. To identify these, a fitting procedure is required. A genetic algorithm was implemented in Python to carry out the fitting process. Genetic algorithms (GA) are robust numerical methods suitable for fitting problems with high parameter counts, non-smooth solution spaces and poor variable separation. While there are many variants of the genetic algorithm, all are based on the idea of a population of candidate solutions which are mutated and then, reproduced if they are relatively 
close to the objective, or culled if they are far. The specifics of the mutation rate, the parametrisation of the function, etc. are chosen depending on the problem. We have chosen a simple approach with a particular mutation strategy, described below, to smooth the search for appropriate solutions.

The fitting of the parameters was achieved using an asexual genetic algorithm with "elitism". This means that the $n$ best solutions are retained after each iteration without mutation thus allowing the "best" genes in the solution pool to be carried forward to the next generation. The objective function is the Euclidean distance between the predicted scrap (from the model) and the tabulated scrap (from the data). The algorithm attempts to minimise this value, while a scoring system based on this distance was used to identify the $n$ best solutions. Convergence was assumed to be reached when the parameters of the best solution were kept largely constant for more than 200 iterations. The population size was 1000 and the elite individuals kept were 500. High mutation rates are imposed at the beginning of the process and subsequently lowered to allow for efficient convergence.

Between each distribution and its successor the fraction $\phi_{i}$ is mutated at each iteration, starting from $t=5$ in the following way:

- Initially, a uniformly distributed variable $v$ between -0.4 and 0.4 was generated

- The variable determined the mutation of the fraction $\phi_{i}$ in the following way:

$$
\phi_{i}=\phi_{i-1}+v \phi_{i-1}
$$

- If the parameters of the best solution were held constant over more than 100 iterations, then the bounds of the uniformly distributed variable $v$ were decreased to -0.2 and 0.2 .

- Finally, as is common in a GA, a crossover function was implemented: with a relatively high probability of $15 \%$, two fractions, picked randomly, are swapped. 
This ensures that large changes are always possible, allowing the whole solution space to be explored.

\subsection{Optimising and Validating the $\mathrm{TF}$}

The result of the GA procedure, is a single TF calibrated over the entire data set, and assuming constant characteristic lifetimes of the products from 1950 to 2014. While lifetimes may well vary over the period, variations are unlikely to be large and are assumed to be within the expected uncertainty. Therefore, the TF provides a single picture of the average lifetimes of all steel product categories, over this period. To verify that the change in lifetimes is in fact small, the optimiser was run over two periods: the first one using scrap from 1950 to 1970 and the second using scrap from 1970 to 2014 .

Identifying a peak corresponding to a single product category in the TF requires two conditions to be met:

- The distributions must be exclusive: it is not possible to distinguish overlapping distributions with the same mean and standard deviation. Overlapping distributions are, therefore, classed as one category.

- The distributions are contiguous: a single product category cannot have a multimodal lifetime probability distribution. If it did, it would be classed as multiple product categories.

To verify that the emergent peaks in the TF are meaningful, an analysis was conducted to compute the contribution of each individual distribution to the overall TF. An arbitrary contribution factor was defined and computed for each distribution at different sampling rates. If this factor was above a defined level of noise, its contribution to an overall peak was classified as meaningful. The contribution factor was determined in the following way.

- The scrap data was de-trended to reveal the discrepancies between the predicted scrap and the scrap data each year. 
- Next, we determined an upper and lower bound between which $90 \%$ of these discrepancies lie.

- We then removed a single distribution from the TF by setting its weighting $\phi$ to 0 and then compute the change in the percentage between the $90 \%$ bounds identified in the previous step. This gives us an idea of the level of contribution of that particular element, to the TF, whereby a large change suggests a large contribution.

- The process was repeated for each element in the TF.

\subsection{Validation}

to validate the overall method, we used disaggregated bottom-up data for the two main steel product categories: construction and vehicles to obtain sector specific lifetime probability distributions. Through doing this, we were able to gain additional insights into the capabilities of the model, validate the overall TF and assess the steel consumption and scrap generation trends in the uK through a socio-economic lens. The following sections explain how the bottom-up data was collected and applied using the methodology described above.

\subsubsection{Predicting arisings from the construction industry}

To compute an estimation of the scrap arisings from the construction industry, we first determined the steel consumed by this sector. We used the methodology outlined in the thesis of ? to estimate the proportion of different types of elements, sections, light sections plates, etc. which go to the construction industry. These proportions, in combination with UK trade and production statistics from the IssB yearbook (?), are then employed to compute the steel inflows to the construction industry over the period from 1899 to 2014.

The corresponding scrap arisings were estimated using this data together with the lifetime probability distributions of buildings. This distribution was determined by inspection of the overall $\mathrm{TF}$; the peak which likely corresponds to construction was 
extracted from the TF and applied to the steel consumption data series, giving the projected scrap arisings. To obtain the probability distribution function from the $\mathrm{TF}$, it was renormalised to reflect the fact that currently (97\%) of the steel from demolition is sent for recycling (?), as indicated by industry surveys. The predicted scrap arising was compared to a limited series of bottom-up data collected by the National Federation of Demolition Contractors (?). More detail on these data series can be found in the appendix.

\subsubsection{Computing the lifetime of vehicles using bottom-up data}

The proposed method is mostly useful when no information is available to create a bottom up model of the material flows. The assess its performance against more commonly used identification procedures, we use the case of the car industry for which detailed information is available.

Using bottom-up data, the magnitude of steel flows into and out of the UK vehicle stock were estimated for each year given that the following quantities were known: the number of new and scrapped vehicles (???), the average mass of new vehicles (??) and the average steel content of new vehicles (?). In general, estimates of the steel scrap output from the vehicle stock is not publicly available and requires creating a stock model to estimate the age distribution of vehicles on the road. Vehicle stock models have been used in past work (???) and generally require knowledge of the age of scrapped vehicles in a given base year. In combination with historic new vehicle registration data, the age of the vehicles at end-of-life was used to compute the lifetime probability distribution for uK vehicles solely from bottom-up data. Applying this to the new vehicle registration data produces an estimate of the scrap output from vehicles. The methodology described in sections 2.1 and 2.3 was then used to compute a PDF which mapped the consumption data approximately to the scrap estimation. This function is equivalent to the lifetime probability distribution of vehicles and was compared to that found using the bottom-up data in order to verify the methodology used. 


\subsubsection{Identifying other steel categories}

The dominant steel product categories are construction and vehicles which account for two thirds of all steel consumption in the UK (?). The remaining third is incorporated into containers, machinery and equipment $e t c$. However, there is a lack of sector-specific data as scrap is rarely disaggregated into product categories with the exception of vehicles and, in recent years, construction. The input data for these two categories were excluded from the aggregated UK data to generate input data corresponding to the steel in other product categories. The same method was applied to scrap data. For construction, where scrap data is only available in recent years, predicted scrap arisings were calculated using the method previously described in section 2.5.1. Once construction and vehicle data was excluded, the genetic algorithm was again applied, as described in section 2.3 , to obtain a PDF describing the lifetime probability distribution of the other product categories.

\section{Results}

UK steel data was used to predict the lifetime probability of all UK steel products over the period 1950-2014. The resulting TF is presented in Figure 2, obtained using both the Weibull and log-normal distributions. There are two distinct peaks in both cases as well as a large 1-year peak represented by the exponential. The first peak, at 9-10 years, most likely relates to vehicles, machinery and equipment. These product categories are expected to have similar lifetimes with overlapping distributions and, therefore, cannot reliably be distinguished. The second peak, at approximately 55 years, most likely correspond to construction. The TF for both distribution types are similar with the dominant peaks occurring at approximately the same characteristic times. Therefore, the use of either distribution is considered acceptable. The predicted scrap output using the Weibull TF compared to the scrap data; a close match is achieved with an average percentage error of $8 \%$, indicating the model is working well.

The parameters of the TF were adjusted between runs to investigate the effect of 
varying the interval between the peaks of adjacent distributions. This varied the number of elements in the summation as well as their characteristic times. Figure 1 illustrates the effect of this variation. As expected, when the discretisation is too fine i.e. 1 or 2 years intervals between the characteristic times $\mu$ (i,e. dark blue curve), there are more fluctuations in the TF and it appears 'noisy'. This is due to the overlap between adjacent peaks being insignificant and so the superposition of adjacent peaks does not yield satisfactory results. On the other hand, when the interval is too wide i.e. 7 or 14 years, the overlap is too large between the peaks and therefore individual peaks cannot be distinguished, leading to a smoothing effect (i.e. yellow curve). Peaks identified in the TF are meaningful and contribute significantly to the scrap prediction. For all sampling rates, the distributions with the highest contributions are at characteristic times of approximately 9-10 years and 50-54 years which correspond to the distinct peaks in Figure 2.

This is reflected in the middle plot of Figure 1, which shows that those peaks are captured robustly with the interval being varied, and therefore should be visible individually when the interval is chosen. Further, the proposed procedure should be robust absent bottom up data, and so have the fewest possible degrees of freedom to fit. Therefore, the choice of the 5-year interval as the value which minimises the number of degrees of freedom in the sampling function, without flattening all the peak is validated.

The optimiser was run separately over two time periods, 1950-70 and 1970-2014, to find possible changes in the characteristic lifetimes of steel products during the time period covered by the uk steel data (Figure 1, bottom). The result shows some minor shift in the dominant peaks, perhaps indicating a slight increase in lifetimes of various steel products. 

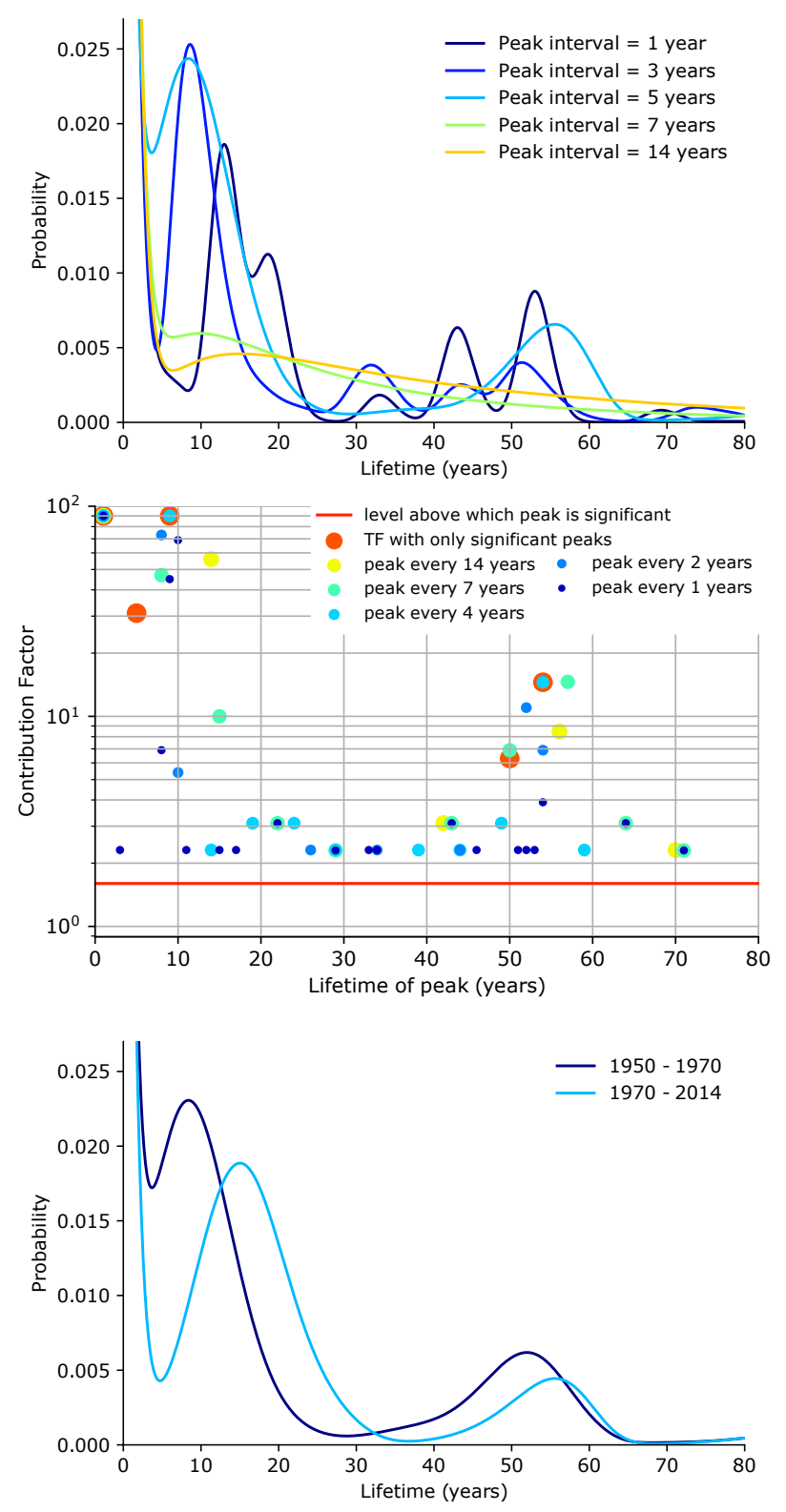

Figure 1: Top. The lifetime probability distribution of steel in the uk between 1950-2014 for different sampling periods i.e. different intervals between the characteristic times for each element in the TF. Middle. The contribution of each element in the TF for different sampling rates. Bottom. The TFS obtained for UK steel for two separate time period: 1950-1970 and 1970-2014. 

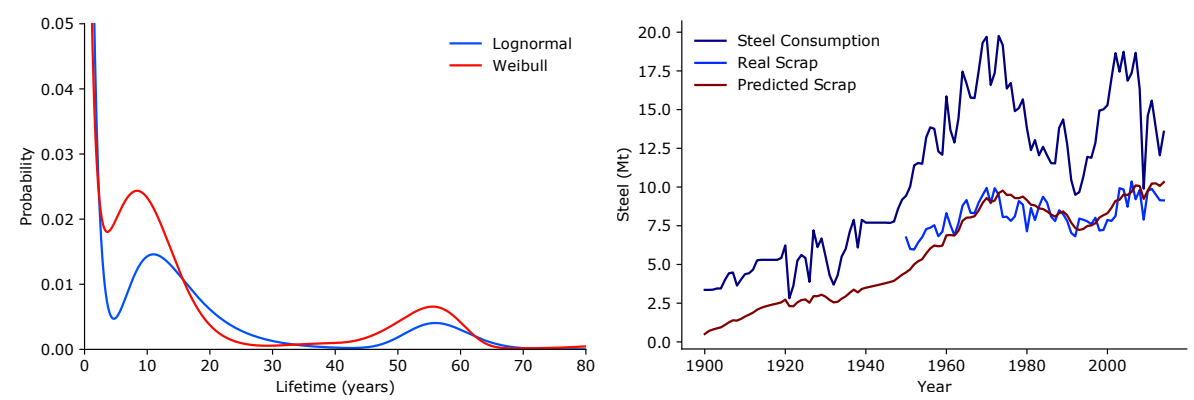

Figure 2: Left. The transfer function of steel in the uK between 1950-2014 using a Weibull and Log-normal distribution. Right. UK steel consumption, scrap generation and the predicted scrap using Weibull between 1950-2014. Underlying data used to create this figure can be found in the Supporting Information.

\subsection{Validating the model using scrap arising data from construction}

The peak corresponding to construction, i.e. at 50-54 years, was extracted from the overall TF shown in Figure 2, as described in Section 2.5.1, using a Weibull tF for simplicity. After re-normalising the extracted function to transform it to a PDF, it was applied to UK construction consumption data (inflows) to predict scrap arisings. Figure 3 shows the predicted scrap arisings, compared to bottom-up data collected by the NFDC, representing $80 \%$ of the top professional demolition contractors by value of contracts (?).

The NFDC survey, shows that 1.07 million metric tons metal was sent for recycling or reuse after demolition, (?) in 2015. The uK annually exports approximately 7 million metric tons steel scrap for recycling (?), as much as the uK consumes annually in a new products (?), representing $78 \%$ of all generated steel scrap in the UK.

\subsection{Validating the model using a bottom-up model of UK vehicles}

The optimisation routine was run using vehicle-specific data from the UK, as described in section 2.5.2. Figure 4 shows the predicted lifetime PDFs obtained for vehicles in our model after normalisation for 1954 and 1980, and the lifetime probability distribution found directly from bottom-up UK data for 1995-2019. The results show an increase in vehicle lifetimes over the 60 -year period covered by the data. The cor- 


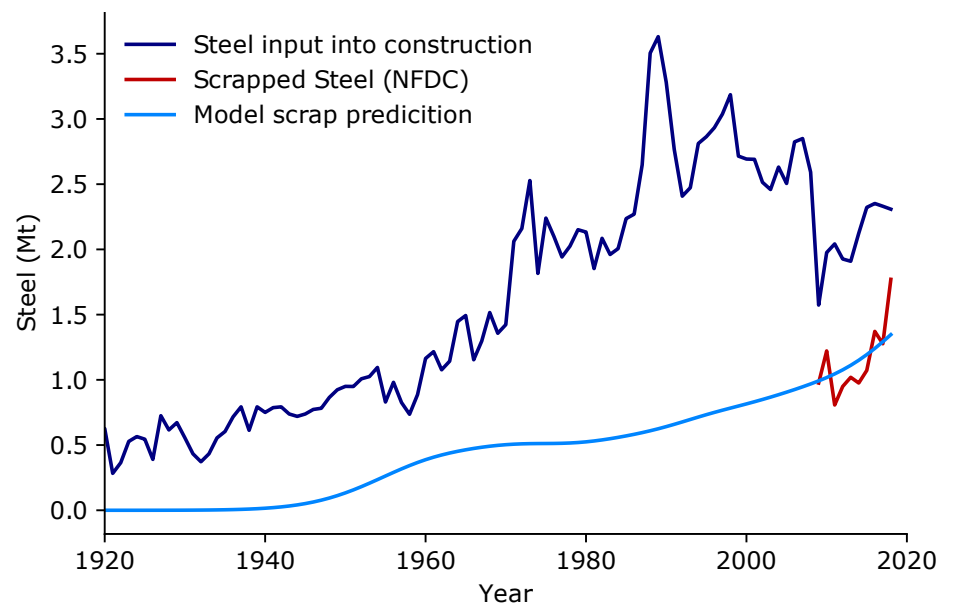

Figure 3: The steel consumption by the construction industry and the predicted scrap arisings compared to bottom up scrap data from the NFDC. Underlying data used to create this figure can be found in the Supporting Information.

responding scrap prediction match closely real vehicle scrap data, with an average percentage error of 5\%. Real vehicle life-spans have increased significantly over the period 1995-2019, but the method proposed in this paper has insufficient temporal resolution to capture this in detail. Further, because the peaks chosen to identify the transfer function are 5-year wide, the fitted transfer function is similarly wider than the real life-time distribution.
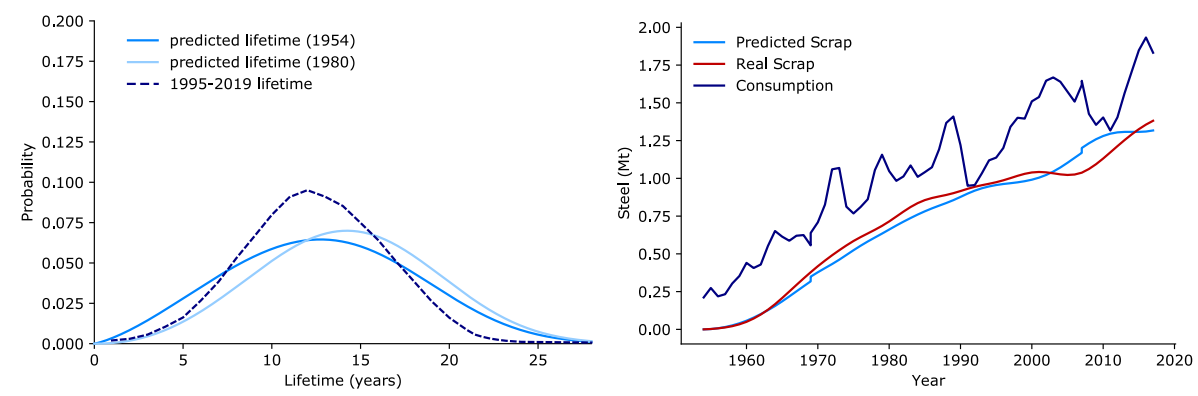

Figure 4: Left. The PDF obtained using the optimisation routine for vehicles in the uK between 1954-1980, and 1980-2018. This is compared to the lifetime probability distributions of vehicles from bottom-up data over this period. A comparable shift is observed as from the bottom-up data. Right. The predicted steel scrap from vehicles corresponding to the PDF compared to steel scrap data and steel consumption by vehicles in the UK (1954-2014). These data can be found in tabular form in the appendix. 


\subsection{Remaining product categories}

A TF was for the remaining steel products (i.e. machinery, containers, equipment, metal goods) was computed by extracting data for vehicles and constriction from the aggregated data, as detailed in section 2.5.3. Figure 5 presents the results and identifies an average lifetime of 20-25 years for all sampling rates.

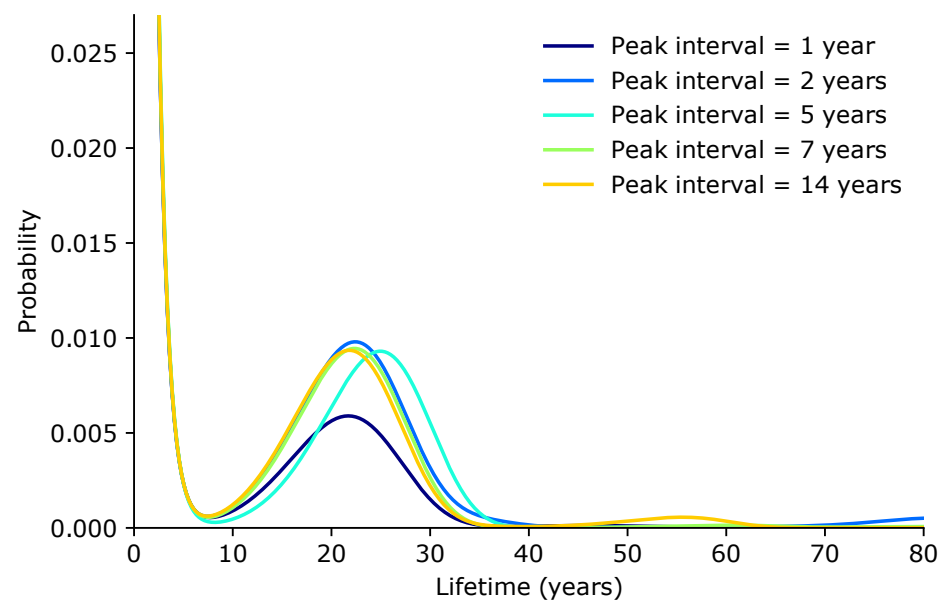

Figure 5: The lifetime probability distribution of steel product categories excluding construction and vehicles in the UK from 1954 to 2014

\section{Discussion}

The modelling strategy and implementation of the method proposed here are straightforward. The results obtained are conserved when the shape of the underlying probability distributions are varied, and the groups of peaks corresponding to product categories remain identifiable across a large range of sampling rates. Nonetheless, a 5 year sampling rate seems to work best, possibly because it best matches the variation of the economic cycle which are not captured in this analysis.

Figure 1 indicates that, while the TF are similar across time periods, there are also key distinctions which suggest increased characteristic times and a slightly higher variance after 1970. Changes in the shape of the TF can be explained by the evolution of 
technological, legal and societal factors over time. For the first peak, the shift is partly attributed to the increase in lifetimes of cars bodies after 1965, due to the introduction of galvanisation, and the end-of-life care directive from the eu which directly manufacturers to make use of common car frames (?). An increase in the quality of durable goods also contributes to increasing lifetimes in the TF. The 1970s Oil Crisis resulted in a significant drop in steel consumption, as can be seen in Figure 2. We assume that this drop in demand was also accompanied by lower demolition and scrappage rates, resulting in increases in the lifetimes of buildings and vehicles respectively. Changing norms within the construction industry may also have contributed to a change in characteristic lifetimes. For example, practice changes in the uK construction market, introduced under the government of Margaret Thatcher, led to a decline in construction activity. These many factors, together, are assumed to have led to an increase in the lifetimes of steel products.

The first peak in Figure 2 is believed to include a range of steel products, including vehicles, equipment, machinery, containers etc.. The corresponding characteristic lifetime is most likely an average of each of these product lifetimes, where the individual distributions overlap to form a single peak. The characteristic lifetime of vehicles from the bottom-up data is slightly higher than the lifetime identified using our method. This can be explained by the wider range of data used in our model, dating back to 1954 , compared to with post-2009 data for actual vehicles. It is known that over this time discrepancy, the introduction of galvanised steel bodies led to a general increase in the longevity of vehicles significantly.

In general, our method is most suitable for the identification of steel product categories with distinct life expectancies, and non-overlapping average lifetimes. When product lifetimes do overlap, bottom-up data can be used to distinguish between overlapping lifetimes. This makes it well suited for the identification of long-lasting product classes such as buildings, provided long data series are available. Despite this limitation, 
the $\mathrm{TF}$ is still robustly representative of the overall lifetime distribution across all steel products and can be used in dynamic material flow analyses predicting future scarp arisings and changes to product stocks.

\section{Conclusion}

We have presented a novel method for the top-down determination of the lifetime distribution of products, which takes as an input trade data, which is of high quality, and widely available for many countries but highly aggregated. A transfer function is identified using a genetic algorithm, and using external information on recycling rates, the transfer function can be, by inspection, transformed to a life-time probability distribution for the classes of product of interest.

Using our proposed method we could identify the life-time of buildings in the uK for which there exists no good bottom-up data. We have compared our results with cars where bottom up data is available, and found that the method found average lifetimes with very good agreement with the bottom-up data, but was less precise when considering the spread of the life-time distribution. Overall, this gives confidence that the method can reliably used when no bottom-up data is available. We hope this new method will make it possible to do wide-ranging, cross-country and time, comparisons of product lifetimes, using only top-down data, while still highlighting important insights into the lifetime distributions of products.

\section{Acknowledgements}

This work was supported EPSRC Material demand reduction: NMZL/112, RG82144, EPSRC reference: EP/N02351X/1 and EPSRC programme grant 'UKFIRES' ref. EP/S019111/1;

\section{References}

ATHENA Institute. Minnesota Demolition Survey: Phase two Report. Technical report,

The ATHENA Institute, 2004. 
Christian Brand. UK Transport Carbon Model Reference Guide Working Paper. UKERC, 2010.

British Standards Institution. BS 7543:1992 - guide to durability of buildings and building elements, products and components. British Standards Institution, 1992.

Daniel R Cooper, Alexandra CH Skelton, Muiris C Moynihan, and Julian M Allwood. Component level strategies for exploiting the lifespan of steel in products. Resources, Conservation and Recycling, 84:24-34, 2014.

Matteo Craglia and Jonathan Cullen. Do technical improvements lead to real efficiency gains? Disaggregating changes in transport energy intensity. Energy Policy, 134 (April):110991, nov 2019. ISSN 03014215. doi: 10.1016/j.enpol.2019.110991. URL https://linkinghub.elsevier.com/retrieve/pii/S0301421519305786.

CRW. Overview of Demolition Waste in the UK. Technical report, CRW, 2008.

Ichiro Daigo, Yuma Igarashi, Yasunari Matsuno, and Yoshihiro Adachi. Accounting for steel stock in japan. ISIJ international, 47(7):1065-1069, 2007.

Ichiro Daigo, Kohei Iwata, Masahiro Oguchi, and Yoshikazu Goto. Lifetime distribution of buildings decided by economic situation at demolition: D-based lifetime distribution. Procedia CIRP, 61:146-151, 2017.

J. Davis, R. Geyer, J. Ley, J. He, R. Clift, A. Kwan, M. Sansom, and T. Jackson. Timedependent material flow analysis of iron and steel in the uk: Part 2. scrap generation and recycling. Resources, Conservation and Recycling, 51(1):118 - 140, 2007. ISSN 0921-3449. doi: https://doi.org/10.1016/j.resconrec.2006.08.007. URL http: //www.sciencedirect.com/science/article/pii/S0921344906001844.

EEF. Key Statistics 2016, UK Steel. Technical report, EEF, 2016. URL http://www2. eef .org.uk/NR/rdonlyres/8DD5EFF5-925E-466D-A3AC-829052D00E7D/ 24665/UKSteelKeyStatsGuide2016.pdf. 
EUROFER. EUROFER survey of NFDC Members. Technical report, EUROFER, July 2012.

A. Gauffin, N. Å. I. Andersson, P. Storm, A. Tilliander, and P. G. Jönsson. Use of volume correlation model to calculate lifetime of end-of-life steel. Ironmaking $\mathcal{E}$ Steelmaking, 42(2):88-96, 2015. doi: 10.1179/1743281214Y.0000000210. URL https ://doi.org/10.1179/1743281214Y.0000000210.

Alicia Gauffin, Nils Å.I. Andersson, Per Storm, Anders Tilliander, and Pär G. Jönsson. Time-varying losses in material flows of steel using dynamic material flow models. Resources, Conservation and Recycling, 116:70 - 83, 2017. ISSN 0921-3449. doi: https://doi.org/10.1016/j.resconrec.2016.09.024. URL http: //wWW.sciencedirect.com/science/article/pii/S0921344916302725.

R. Gyllenram, S. Ekerot, and P. Jönsson. Lubricating the recycling machine. Revue Métall, 109(5):349—358, 2012.

Satu Huuhka and Jukka Lahdensivu. Statistical and geographical study on demolished buildings. Building Research $\mathcal{F}$ Information, 44(1):73-96, January 2016. ISSN 0961-3218, 1466-4321. doi: 10.1080/09613218.2014.980101. URL http://www . tandf online.com/doi/full/10.1080/09613218.2014.980101.

IEA. Perspectives for the Clean Energy Transition - The critical role of buildings. IEA, 2019, www.iea.org/publications/reports/ PerspectivesfortheCleanEnergyTransition/.

IEA. Global Status Report 2018 - Towards a zero-emission, efficient, and resilient buildings and construction sector, Global Alliance for Building and Construction and International Energy Agency. Technical report, Global Alliance for Building and Construction, International Energy Agency, 2018. 
Yuma Igarashi, Ichiro Daigo, Yasunari Matsuno, and Yoshihiro Adachi. Dynamic material flow analysis for stainless steels in japan-reductions potential of $\mathrm{CO} 2$ emissions by promoting closed loop recycling of stainless steels. ISIJ International, 47(5): 758-763, 2007. ISSN 0915-1559, 1347-5460. doi: 10.2355/isijinternational.47. 758. URL http://joi.jlc.jst.go.jp/JST.JSTAGE/isijinternational/ 47.758? from=CrossRef.

ISSB. Annual Statistics - UK Iron \& Steel Industry. Technical report, ISSB Limited, 2015.

Shigemi Kagawa, Keisuke Nansai, Yasushi Kondo, Klaus Hubacek, Sangwon Suh, Jan Minx, Yuki Kudoh, Tomohiro Tasaki, and Shinichiro Nakamura. Role of motor vehicle lifetime extension in climate change policy, 2011.

Yukio Komatsu. Report of an investigation of the life-time distribution of japanese houses in 1987 (in japanese). J Archit Plann Environ Eng AIJ, 439:101-110, 1992.

Fridolin Krausmann, Dominik Wiedenhofer, Christian Lauk, Willi Haas, Hiroki Tanikawa, Tomer Fishman, Alessio Miatto, Heinz Schandl, and Helmut Haberl. Global socioeconomic material stocks rise 23 -fold over the 20th century and require half of annual resource use. Proceedings of the National Academy of Sciences, 114 (8):1880-1885, 2017.

James Ley. An environmental and material flow analysis of the UK steel construction sector. PhD Thesis, University of Wales, Cardiff University, 2003.

Niall P. D. Martin, Justin D.K. K Bishop, and Adam M. Boies. How Well Do We Know the Future of CO 2 Emissions? Projecting Fleet Emissions from Light Duty Vehicle Technology Drivers. Environmental Science E Technology, page acs.est.6b04746, 2017. ISSN 0013-936X. doi: 10.1021/acs.est.6b04746. URL http://pubs.acs.org/doi/abs/10.1021/acs.est.6b04746. 
Zak McGregor. www.carfolio.com, 2017.

Daniel B Müller. Stock dynamics for forecasting material flows—case study for housing in the netherlands. Ecological Economics, 59(1):142-156, 2006.

Daniel B. Müller, Matthew Altonji Jing Cao, Elif Kongar, Paul-Harvey Weiner, and T.E. Graedel. Service lifetimes of mineral end uses. US Geological Survey (USGS), 2007.

Esther Müller, Lorenz M. Hilty, Rolf Widmer, Mathias Schluep, and Martin Faulstich. Modeling metal stocks and flows: a review of dynamic material flow analysis methods. Environmental science $\mathcal{E}$ technology, 48 4:2102-13, 2014.

Yuya Nakamoto, Daisuke Nishijima, and Shigemi Kagawa. The role of vehicle lifetime extensions of countries on global co2 emissions. Journal of cleaner production, 207: 1040-1046, 2019.

Shinichiro Nakamura, Yasushi Kondo, Shigemi Kagawa, Kazuyo Matsubae, Kenichi Nakajima, and Tetsuya Nagasaka. Matrace: Tracing the fate of materials over time and across products in open-loop recycling. Environmental science $\mathcal{E}$ technology, 48 (13):7207-7214, 2014.

NFDC. NFDC waste survey. Technical report, National Federation of Demolition Contractors, 2015.

Masahiro Oguchi and Masaaki Fuse. Regional and longitudinal estimation of product lifespan distribution: a case study for automobiles and a simplified estimation method. Environmental science $\mathcal{E}$ technology, 49(3):1738-1743, 2015.

Stefan Pauliuk, Tao Wang, and Daniel B Müller. Moving toward the circular economy: The role of stocks in the chinese steel cycle. Environmental science $\mathcal{E}$ technology, 46 (1):148-154, 2012. 
Stefan Pauliuk, Tao Wang, and Daniel B. Müller. Steel all over the world: Estimating in-use stocks of iron for 200 countries. Resources, Conservation and Recycling, 71: $22-30,2013$.

Stefan Pauliuk, Yasushi Kondo, Shinichiro Nakamura, and Kenichi Nakajima. Regional distribution and losses of end-of-life steel throughout multiple product life cycles-insights from the global multiregional matrace model. Resources, Conservation and Recycling, 116:84-93, 2017.

Michael Sansom and Nicholas Avery. Briefing: Reuse and recycling rates of uk steel demolition arisings. Proceedings of the Institution of Civil Engineers - Engineering Sustainability, 167(3):89-94, 2014a. doi: 10.1680/ensu.13.00026. URL http: //dx.doi.org/10.1680/ensu.13.00026.

Michael Sansom and Nicholas Avery. Briefing: Reuse and recycling rates of uk steel demolition arisings. Proceedings of the Institution of Civil Engineers - Engineering Sustainability, 167:89-94, 06 2014b. doi: 10.1680/ensu.13.00026.

André Cabrera Serrenho, Jonathan B Norman, and Julian M Allwood. The impact of reducing car weight on global emissions: the future fleet in Great Britain. Philosophical Transactions of the Royal Society A: Mathematical, Physical and Engineering Sciences, 2017.

Alexandra CH Skelton and Julian M Allwood. Product life trade-offs: what if products fail early? Environmental science $\mathcal{E}$ technology, 47(3):1719-1728, 2013.

UK Department for Transport. Table Veh0103: Licensed vehicles at the end of the year by tax class, 2019a.

UK Department for Transport. Table Veh0153: Vehicles registered or the first time by tax class, 2019b. 
UK Department for Transport. Table Veh0124: Licensed cars by make, model and year of first registration, 2019c.

UNFCCC. Report of the conference of the parties on its twenty-first session, held in paris from 30 november to 13 december 2015 addendum contents part two: Action taken by the conference of the parties at its twenty-first session. Decision 1/CP. 21 Adoption of the Paris Agreement, 2015.

United Nations. World population prospects: the 2017 revision, key findings and advance tables. United Nations, New york, 2017.

World Steel Association. World Steel in Figures 2017, World Steel Association. Technical report, World Steel Association, 2017. 